\title{
Duckling Falcon and SaaS Applications
}

\author{
Kejun Dong ${ }^{1}$ \\ Computern Network Information Center, Chinese Academy of Sciences \\ 4 Zhongguancun Nansijie, Haidian, Beijing China \\ E-mail: kevinecstnet.cn
}

\section{Kai Nan}

Computern Network Information Center, Chinese Academy of Sciences

4 Zhongguancun Nansijie, Haidian, Beijing China

E-mail: nankai@cstnet. cn

\section{Ji Li}

Computern Network Information Center, Chinese Academy of Sciences

4 Zhongguancun Nansijie, Haidian, Beijing China

E-mail: Iijiecstnet. cn

Duckling is a collaboration environment to support e-science applications in Chinese Academy of Sciences(CAS), as well as falcon, which is a cloud functionality of Duckling, to enable Software as a Service applications, for its robustness and scalability. Duckling Falcon is also a common component as part of CAS Science Cloud Platform, which is a multiple cloud service for researchers around CAS.

Speaker 


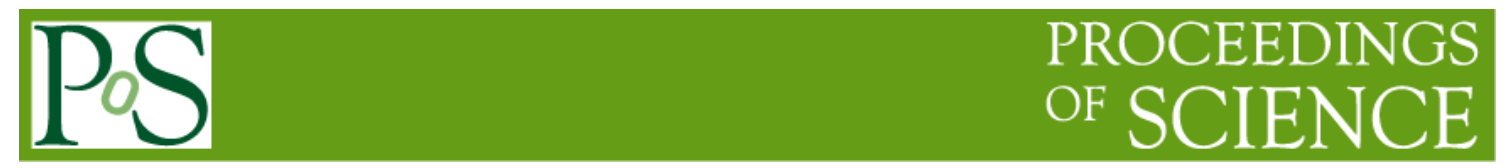

e-Infrastructures for e-Sciences 2013 A CHAIN-REDS Workshop organised under the aegis of the European Commission (eIeS 2013)

October 22, 2013

Beijing, P.R. of China 


\section{Introduction}

Rapid advances in scientific research collaboration have led to significant challenges in promoting service performance to build, test, deploy and scale SaaS (Software as a Service) applications. This poster presents a Platform as a Service (PaaS) environment which integrates Duckling falcon platform and Cloud Foundry open source software.

The Duckling Collaboration Environment, developed by Computer Network Information Center (CNIC), Chinese Academy of Sciences (CAS), is a comprehensive resource sharing and collaboration platform to support e-science applications in CAS. There are two functionalities of Duckling Collaboration Environment, including the cloud open platform named falcon, and the online cloud services/applications named "Research Online" at http://www.escience.cn.

The presentation introduced the Duckling Falcon Platform, as well as the SaaS applications based on the duckling falcon, as a web portal named "Research Online". A scenario is presented to extend the "Site Navigation" application, from one VM to $30 \mathrm{VMs}$, to show the scalability of Duckling falcon and Cloud Foundry with high service performance. And a performance test and evaluation will also be showed in this scenario.

\section{Falcon and Cloud Foundry}

Falcon is a cloud functionality of Duckling, to enable SaaS applications, for its robustness and scalability. And we also integrate Cloud Foundry, which is an open source Platform as a Service (PaaS) project (http://www.cloudfoundry.com/) to provide a faster and easier way to build, test, deploy and scale applications.

Cloud Foundry supports applications written in several JVM-based languages, including Java, Groovy, and Scala, Ruby applications written using the Rails, Sinatra, and Rack frameworks and Javascript applications using the Node.js, as well as most of frameworks including Spring, Grails, Play, Lift, and so on.

\begin{tabular}{|c|c|c|}
\hline Functionality & Falcon & Cloud Foundry \\
\hline App Auto Deployment & $\times$ & $\mathrm{V}$ \\
\hline Web High Availability & $\mathrm{v}$ & $\mathrm{V}$ \\
\hline Multiple Language Support (Java/Python/...) & $\times$ & $\mathrm{V}$ \\
\hline Service High Availability (MYSQL/MongoDB/...) & $\mathrm{V}$ & $\times$ \\
\hline Session Stateless (Memcached) & $\mathrm{V}$ & $\times$ \\
\hline App Instance Migration & $\mathrm{V}$ & $\mathrm{V}$ \\
\hline
\end{tabular}

\section{Demo Case and Evaluation}

In the demo case, a falcon-enabled application named "SiteNav" is deployed in the experiment. A 5/10/20 instances are used as the load balance web container cluster for the SiteNav application. The siege tool is used to simulate the concurrent of web browser. We may 
get results from the demo case that all visits are load balanced to the server instances with the fault tolerant support. And figure 3 shows the response time of the Siege test. 100-400 stands for the concurrent threads for the test and we can draw the conclusion that we can easily extend the cluster from 5 instances to 20 instances in few seconds by falcon with cloud foundry support and get the better response time.

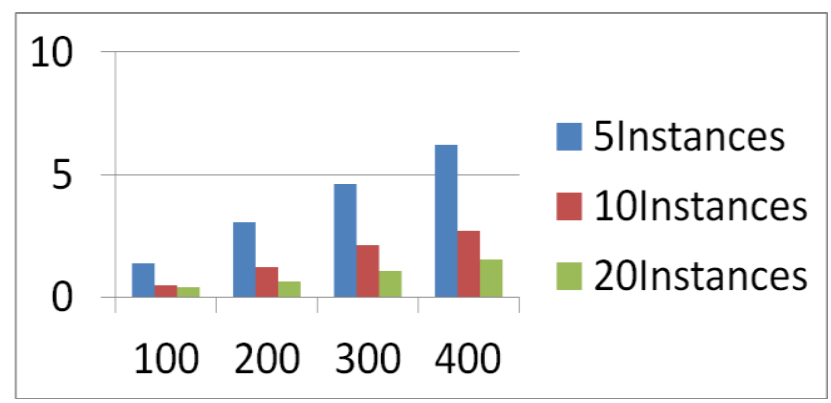

\subsection{SaaS Applications}

Duckling which is a collaborative work environment package independently developed by the CSTNET is a set of Internet-based tools and application development platform. The duration of 2012, based on Duckling platform, had developed the research online SaaS products (Research Online, http://www.escience.cn), which include DDL, CSP, dHome, and so on, to achieve the application platform pattern of development. So far, passport users of Duckling platform exceeded 248 thousands people.

DDL, stands for team documentation library, is a socialized collaboration platform for scientific research teams. It not only helps research teams to efficiently share ideas, documents and charts, but also provides a mobile client for viewing and sharing information anytime and anywhere. Up to October 2013, users of research online document library amounted to 27000 people, 2494 teams.

CSP, stands for Conference Services Platform (CSP, http://csp.escience.cn), provides a one-stop information platform and a comprehensive conference website for construction and management. Up to October, 2013, there were more than 59,000 conference participants registered, and more than 700 conferences which covered $85 \%$ of institutes, and $99.9 \%$ of the service availability.

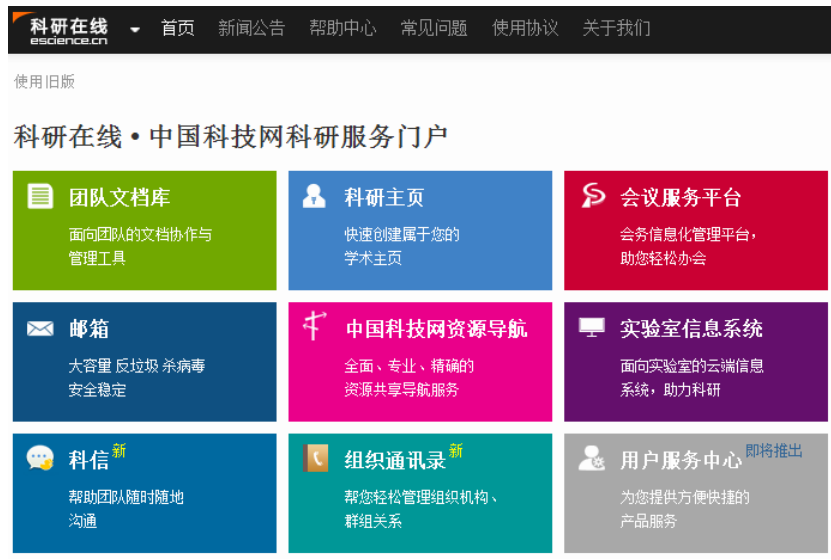




\section{References}

[1] K. Dong, K. Nan, S. Tilak, C. Zheng, D. Xu, J. Schulze, P. Arzberger, W. Li, "Real tIme bioMEdical data Streaming platform (RIMES): A data-intensive virtual environment," CSO 2010, pp. 342-346, 2010

[2] J. Yu, K. Dong, K. Nan, "Duckling: Towards cloud service for scientific collaboration system," CSCW2012, pp. 259-262, Feb. 2012.

[3] J. Yu, Y. Di, K. Dong, K. Nan, "Research Online: Cloud Service Platform for Internet Collaborative Environment," Journal of Huazhong University of Science and Technology (National Science Edition) Vol.39 Sup. I., pp. 33-37, June 2011.

[4] K. Nan, K. Dong, J. Xie, J. Yu, "Research collaboration platform for cloud service,” Journal of Huazhong University of Science and Technology (National Science Edition) Vol.38 Sup. I., pp. 1419, June 2010.

[5] K. Nan, K. Dong, J. Xie, D. Yang, J. Yu, "VLAB: An e-Science Collaboration Framework for CAS Scientists," CODATA 2008, Springer Press, 2008.

[6] X. Su, Y. Ma, H. Yang, X. Chang, K. Nan, J. Xu, K. Ning, “An open-source collaboration environment for metagenomics research," eScience 2011, pp. 7-14, December 2011. 